\section{P64 COMPARISON OF SOCIOECONOMIC DIFFERENCES IN HEALTH USING OBJECTIVE AND SELF-REPORTED MEASURES OF THE SAME CONDITION: EVIDENCE FROM THE HEALTH SURVEY FOR ENGLAND}

${ }^{1}$ JS Mindell*, ${ }^{2}$ S Morris, ${ }^{2}$ A Conolly, ${ }^{1} S$ Scholes. ${ }^{1}$ Epidemiology and Public Health, University College London, London, UK; ${ }^{2}$ Health, National Centre for Social Research, London, UK

10.1136/jech-2017-SSMAbstracts. 165

Background Studies quantifying health inequalities typically rely on self-report data. Systematic differences in reporting error (participants denying, minimising, or being unaware of their condition) could result in inaccurate estimates of inequalities. Using Health Survey for England data (2012-2014), we compared the relationship between socioeconomic status (SES) and health using objective and contemporaneous self-reported measures of the same condition.

Methods Our study focused on four outcomes: obesity $(n=19,993)$, raised blood pressure $(n=14,398)$, diabetes $(\mathrm{n}=12,248)$, and hearing $(\mathrm{n}=5,334)$. Obesity $\left(\mathrm{BMI} \geq 30 \mathrm{~kg} / \mathrm{m}^{2}\right)$ using interviewer-measured height and weight was compared with self-reported height and weight. Blood pressure (BP) measurements (raised BP: systolic/diastolic BP $\geq 140 / 90 \mathrm{mmHg}$ ) were compared with high BP reported as a longlasting illness/ condition. Values of glycated haemoglobin (diabetes: $\geq 6.5 \%$ ) were compared with reported diabetes including hyperglycaemia as a longlasting illness/condition. Using an audiometric screening test, hearing loss was defined as impairment in the better hearing ear to the level of $\geq 35 \mathrm{dBHL}$ at $3 \mathrm{kHz}$; selfreported hearing difficulty was a perceived hearing difficulty, or report of current hearing-aid use. Equivalised household income was our chosen indicator of SES. Logistic regression (using Stata) was used to examine the statistical significance of SES differences. We present the age-adjusted Odds Ratios (AOR) with 95\% Confidence Intervals (CI) separately for the objective and self-report measures comparing participants in the lowest income quintile with those in the highest.

Results Except for hearing, self-reported estimates were lower than those using objective data. For example, 25.3\% (95\%CI: $24.2 \%-26.3 \%$ ) of men and $24.1 \%$ (95\%CI: $23.2 \%-25.1 \%$ ) of women were obese using interviewer-measured BMI; $18.9 \%$ (95\%CI: $18.0 \%-19.9 \%$ ) of men and 18.1\% (95\%CI: $17.2 \%-$ $18.9 \%)$ of women were obese based on self-report.

Age-adjusted odds of interviewer-measured obesity (AOR: 1.64; 95\% CI: 1.44-1.87, p<0.001) and self-reported obesity $(1.69 ; 1.47-1.95, \mathrm{p}<0.001)$ were significantly higher for participants in the lowest income quintile (reference: highest quintile). Findings for diabetes were similar. We found that the magnitude of inequalities for raised BP and for hearing varied by whether self-reported or objective data was used. AORs for each raised BP measure did not attain significance among men. AORs for women were higher for objectivelymeasured raised BP $(1.53 ; 1.19-1.97, \mathrm{p}<0.001)$ than for selfreport $(1.37,0.97-1.94, p=0.075)$. AORs for low income men were higher using the audiometric screening test (1.81; 1.14-2.89, $\mathrm{p}=0.012$ ) but not for self-reported hearing problems $(0.92,0.63-1.34, p=0.657)$. A similar but weaker pattern was found for women.

Conclusion Reliance on self-reported data, particularly for asymptomatic conditions such as raised BP, might underestimate socioeconomic differences in health.

\section{P65 SOCIOECONOMIC DIFFERENCES IN HEARING AMONG MIDDLE-AGED AND OLDER ADULTS IN THE HEALTH SURVEY FOR ENGLAND}

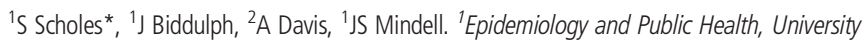
College London, London, UK; ${ }^{2}$ UCL Ear Institute, University College London, London, UK

\subsection{6/jech-2017-SSMAbstracts. 166}

Background Hearing loss impacts on physical and social functioning. Using audiometry data collected in 2014, we estimated the current prevalence of hearing loss in a nationallyrepresentative sample of adults aged 45 +years. We also estimated current hearing aid use among persons with hearing loss.

Methods Cross-sectional analysis of the Health Survey for England 2014 ( $n=3292$ participants aged 45+years with valid screening audiometry data; $\mathrm{n}=769$ with hearing loss). Using Stata, we estimated the prevalence of: (1) hearing loss (defined at $\geq 35 \mathrm{dBHL}$ at $3 \mathrm{kHz}$ in the better hearing ear), and (2) current hearing aid use (among persons with hearing loss). Differences in these outcomes were examined by groups stratified by demography, duration of work-related noise exposure, cardiovascular disease (CVD) risk factors, and socioeconomic status (SES: equivalised household income, education, and area-based Index of Multiple Deprivation). Using sex-specific logistic regression modelling, we evaluated the associations between SES and hearing after adjustment for potential confounders. Results are presented as fully-adjusted Odds Ratios (OR) with 95\% Confidence Intervals (95\% CIs).

Results Hearing loss was higher for men (26\%) than women $(20 \%, \mathrm{p}<0.001)$; increased monotonically with age; and was higher for men exposed to work-related noise for $\geq 5$ years. SES differences in the odds of hearing loss remained significant in fully-adjusted models for men but not for women. For example, the odds of hearing loss were almost twice as high for men in the lowest versus the highest income tertile (OR: 1.77; 95\% CI: 1.15-2.74).

Among persons with hearing loss, the prevalence of current hearing aid use was similar for men (30\%) and women $(27 \%$, $\mathrm{p}=0.533)$; increased with the severity of hearing loss; and increased monotonically with age but remained below $40 \%$ even for persons aged $\geq 75$ years. Fully-adjusted ORs for current hearing aid use were lower for persons in the lower SES groups among men but not among women, but SES differences did not attain statistical significance. For example, the odds of current hearing aid use for men in the lowest versus the highest income tertile were 0.55 (95\% CI: 0.27-1.11).

Conclusion Hearing loss is another potential source of socioeconomic inequalities in health, especially in men. Among persons aged $\geq 45$ years, more than one in four men and one in five women had hearing loss severe enough to benefit from hearing aid use. However, there is significant unmet need: fewer than one-third of adults with hearing loss currently used a hearing aid. 